\title{
Resolution of curvature singularities from quantum mechanical and loop perspective
}

\author{
T. Tahamtan ${ }^{1, \mathrm{a}}$, O. Svítek ${ }^{2, \mathrm{~b}}$ \\ ${ }^{1}$ Department of Physics, Eastern Mediterranean University, G. Magusa, North Cyprus, Mersin 10, Turkey \\ ${ }^{2}$ Faculty of Mathematics and Physics, Institute of Theoretical Physics, Charles University in Prague, V Holešovičkách 2 , \\ 18000 Prague 8, Czech Republic
}

Received: 31 December 2013 / Accepted: 21 July 2014 / Published online: 7 August 2014

(C) The Author(s) 2014. This article is published with open access at Springerlink.com

\begin{abstract}
We analyze the persistence of curvature singularities when analyzed using quantum theory. First, quantum test particles obeying the Klein-Gordon and ChandrasekharDirac equation are used to probe the classical timelike naked singularity. We show that the classical singularity is felt even by our quantum probes. Next, we use loop quantization to resolve a singularity hidden beneath the horizon. The singularity is resolved in this case.
\end{abstract}

\section{Introduction}

One of the important predictions of the Einstein theory of general relativity is the formation of spacetime singularities. In classical general relativity, singularities are defined as points in which the evolution of timelike or null geodesics is not defined after a finite proper time. According to the classification of the classical singularities devised by Ellis and Schmidt [1], scalar curvature singularities are strongest in the sense that the spacetime possesses incomplete geodesics ending in them and all the physical quantities such as the gravitational field (scalars formed from the curvature tensor), energy density, and tidal forces diverge at the singular point.

But such divergence of physical quantities signifies the breakdown of the predictive power of classical general relativity. If these singularities are covered by a horizon (as supposed by the Cosmic Censorship Conjecture) then at least the physically most relevant region of spacetime is under control. Naked singularities (those not covered by a horizon), on the other hand, provide an observer with causal access to the region of diverging quantities and this should be avoided. However, even singularities covered by the horizon can be

\footnotetext{
a e-mail: tayabeh.tahamtan@emu.edu.tr

b e-mail: ota@matfyz.cz
}

accessed by an in-falling observer and, more importantly, we would like to have a theory that lacks divergences, at least effectively.

The natural direction for resolving the problem of singularities in classical theory is in investigating their persistence in the quantum picture. Although we do not have a final quantum theory of gravity we still have several tools for analyzing quantum singularities. The first approach relies on examining the properties of quantum particle wave functions on the background represented by the studied geometry. This is a frequently used technique based on well understood properties of operators on a Hilbert space. To move further, one might proceed to using quantum fields and possibly even the backreaction of the background geometry using semiclassical Einstein equations with a suitably regularized stress energy tensor. Finally, one can apply quantization of the geometry itself. The last approach is in principle the most precise but relies on the selected quantization method and we have no generally accepted one in the case of gravity.

Quantum singularities were studied for different specific situations (and using also generalizations), mainly using the first approach [2-15]. Recently, singularities in $f(R)$ gravity were investigated in the presence of a linear electromagnetic field [16].

We will apply two of the above mentioned approaches for analysis of the singularity in the case of the general metric of a global monopole [17-19], which is determined by two parameters - one characterizing the "Schwarzschildtype mass" and the other one the deficit of the solid angle. The singularity is generally covered by a single horizon but the class of metrics also contains, as a special case, a naked singularity, which is analyzed from the quantum mechanical point of view using the technique of Horowitz and Marolf [20] (who continued the pioneering work of Wald [21]). This method for analyzing timelike singularities is based on the investigation of self-adjoint extensions of the evolution 
operator associated with the given wave equation. If it is unique the spacetime is deemed quantum mechanically nonsingular. The analysis is carried out for relativistic quantum particle wave equations on a fixed background. Specifically, we review the previous results for the Klein-Gordon equation and show the calculation using the Newman-Penrose formalism for the Dirac equation, both in the case of a pure global monopole with a naked singularity for which the method was developed.

But as already mentioned, the most reliable method when trying to investigate the possible removal of the singularities from the geometry is quantum gravity. Here we have selected the loop quantization method inspired by [22-24], where the spacetime beneath the horizon (in the non-naked subclass) is isometric to the Kantowski-Sachs cosmology. Then one can apply the methods from Loop Quantum Cosmology (LQC), which are based on loop quantization on the restricted configuration space. In this way, the results for the resolution of the initial cosmological singularity are translated to statements about the singularity at the origin, $r=0$.

\section{The general metric for global monopole}

It is well known that different types of non-standard topological objects may have been formed during the initial evolution of the Universe, such as domain walls, cosmic strings, and monopoles [17-19,25]. The basic idea is that these topological defects have formed as a result of the breakdown of local or global gauge symmetries. The simplest model that gives rise to a global monopole is described by the Lagrangian

$L=\frac{1}{2} \partial_{\mu} \phi^{a} \partial^{\mu} \phi^{a}$,

where $\phi^{a}$ is a triplet of scalar fields, $a=1,2,3$. The model has a global $O(3)$ symmetry, which is spontaneously broken to $U(1)$. The field configuration describing the monopole is

$\phi^{a}=\eta \frac{x^{a}}{r}$

where $x^{a} x^{a}=r^{2}$. We assume that the underlying geometry is general static spherically symmetric and is described by the line element

$\mathrm{d} s^{2}=-B(r) \mathrm{d} t^{2}+\frac{\mathrm{d} r^{2}}{A(r)}+r^{2}\left(\mathrm{~d} \theta^{2}+\sin ^{2} \theta \mathrm{d} \phi^{2}\right)$,

with the usual relation between the spherical coordinates, $r, \theta, \phi$, and the Cartesian coordinates, $x^{a}$. The Lagrangian for the field configuration given above simplifies in the following way:

$L=\frac{1}{2}\left(\partial_{\theta} \phi^{a} \partial^{\theta} \phi^{a}+\partial_{\phi} \phi^{a} \partial^{\phi} \phi^{a}\right)=\frac{\eta^{2}}{r^{2}}$, and the diagonal energy momentum tensor is given by these components

$T_{t}^{t}=T_{r}^{r}=-\frac{\eta^{2}}{r^{2}}, \quad T_{\theta}^{\theta}=T_{\phi}^{\phi}=0$.

The general solution of the Einstein equations with this $T_{\mu}^{\nu}$ is

$B=A=1-8 \pi G \eta^{2}-\frac{2 G M}{r}$

where $M$ is a constant of integration. The metric describes a black hole of mass $M$, carrying a global monopole charge characterized by $\eta$. Such a black hole can be formed if a global monopole is swallowed by an ordinary black hole [17-19].

The Kretschmann scalar which indicates the formation of a curvature singularity is given by

$\mathcal{K}=\frac{48 M^{2} G^{2}}{r^{6}}+\frac{128 M \pi G^{2} \eta^{2}}{r^{5}}+\frac{256 \pi^{2} G^{2} \eta^{4}}{r^{4}}$.

It is obvious that $r=0$ is a typical central curvature singularity (scalar curvature singularity according to above mentioned classification) and the dominant contribution comes from the term corresponding to the black hole mass $M$. If $M>0$ the singularity is evidently spacelike and covered by a single horizon.

\section{Global monopole and its singularity}

If we assume that the mass term is negligible on the astrophysical scale or vanishing, we will have

$\mathrm{d} s^{2}=-\left(1-8 \pi G \eta^{2}\right) \mathrm{d} t^{2}+\frac{\mathrm{d} r^{2}}{\left(1-8 \pi G \eta^{2}\right)}+r^{2} \mathrm{~d} \Omega^{2}$.

For simplicity we choose $\alpha^{2}=1-8 \pi G \eta^{2}$ and by rescaling the $r$ and $t$ variables, we can rewrite the monopole metric as

$\mathrm{d} s^{2}=-\mathrm{d} t^{2}+\mathrm{d} r^{2}+\alpha^{2} r^{2}\left(\mathrm{~d} \theta^{2}+\sin ^{2} \theta \mathrm{d} \varphi^{2}\right)$.

If we calculate the Kretschmann scalar,

$\mathcal{K}=4 \frac{\left(\alpha^{2}-1\right)^{2}}{r^{4} \alpha^{4}}$,

still there is a weaker singularity at $r=0$. From the metric (7) one can immediately see that the singularity is timelike. This time, because our simplified metric does not have the horizon, the singularity is naked.

\section{Naked singularity}

As mentioned in the Introduction a naked singularity poses serious problems and its resolution would be desirable. In 
this section, the occurrence of naked singularities in the case of a global monopole will be analyzed from the quantum mechanical point of view. In probing the singularity, quantum test particles obeying the Klein-Gordon and Dirac equations are used. The reason for using two different types of fields is to clarify whether the classical singularity is sensitive to the spin of the fields.

According to Horowitz and Marolf (HM) [20], the singular character of the spacetime is defined as the ambiguity in the evolution of the wave functions. That is to say, the singular character is determined based on the number of self-adjoint extensions of the evolution operator to the entire Hilbert space. If the extension is unique, it is said that the spacetime is quantum mechanically regular. A brief review of the method follows.

Consider a static spacetime $\left(\mathcal{M}, g_{\mu \nu}\right)$ with a timelike Killing vector field $\xi^{\mu}$. Let $t$ denote the Killing parameter and $\Sigma$ denote a static slice. The Klein-Gordon equation in this space is

$$
\left(\nabla^{\mu} \nabla_{\mu}-M^{2}\right) \psi=0 .
$$

This equation can be written in the form

$$
\frac{\partial^{2} \psi}{\partial t^{2}}=\sqrt{f} D^{i}\left(\sqrt{f} D_{i} \psi\right)-f M^{2} \psi=-A \psi,
$$

in which $f=-\xi^{\mu} \xi_{\mu}$ and $D_{i}$ is the spatial covariant derivative on $\Sigma$. We assume that the Hilbert space $\mathcal{H}=L^{2}(\Sigma, \mu)$ is the space of square integrable functions on $\Sigma$ with appropriate measure $\mu$. Initially the operator $A$ is defined on smooth functions with compact support $C_{0}^{\infty}(\Sigma)$. Since the operator $A$ is real, positive, and symmetric, its self-adjoint extensions always exist. If it has a unique extension $A_{E}$, then $A$ is called essentially self-adjoint [26-28]. Accordingly, the Klein-Gordon equation for a free particle satisfies

$i \frac{\mathrm{d} \psi}{\mathrm{d} t}=\sqrt{A_{E}} \psi$,

with the solution

$\psi(t)=\exp \left[-i t \sqrt{A_{E}}\right] \psi(0)$.

If $A$ is not essentially self-adjoint, the future time evolution of the wave function (12) is ambiguous. Then the HM criterion defines the spacetime as quantum mechanically singular. However, if there is only a single self-adjoint extension, the operator $A$ is said to be essentially self-adjoint and the quantum evolution described by (12) is uniquely determined by the initial conditions. According to the HM criterion, this spacetime is said to be quantum mechanically non-singular. In order to determine the number of self-adjoint extensions, the concept of deficiency indices is used. The deficiency subspaces $N_{ \pm}$are defined by (see Ref. [29] for a detailed math- ematical background)

$$
\begin{aligned}
N_{+}= & \left\{\psi \in D\left(A^{*}\right), A^{*} \psi=Z_{+} \psi, \operatorname{Im} Z_{+}>0\right\} \\
& \text { with dimension } n_{+} \\
N_{-}= & \left\{\psi \in D\left(A^{*}\right), A^{*} \psi=Z_{-} \psi, \operatorname{Im} Z_{-}<0\right\} \\
& \text { with dimension } n_{-} .
\end{aligned}
$$

The dimensions $\left(n_{+}, n_{-}\right)$are the deficiency indices of the operator $A$. The indices $n_{+}\left(n_{-}\right)$are completely independent of the choice of $Z_{+}\left(Z_{-}\right)$, depending only on whether $Z$ lies in the upper (lower) half complex plane. Generally one takes $Z_{+}=i \lambda$ and $Z_{-}=-i \lambda$, where $\lambda$ is an arbitrary positive constant necessary for dimensional reasons. The determination of deficiency indices then reduces to counting the number of solutions of $A^{*} \psi=Z \psi$ (for $\lambda=1$ ),

$A^{*} \psi \pm i \psi=0$

that belong to the Hilbert space $\mathcal{H}$. If there are no square integrable solutions (i.e. $n_{+}=n_{-}=0$ ), the operator $A$ possesses a unique self-adjoint extension and it is essentially self-adjoint. Consequently, a sufficient condition for the operator $A$ to be essentially self-adjoint is to find only solutions satisfying (14) that do not belong to the Hilbert space.

\subsection{Klein-Gordon fields}

The Klein-Gordon equation for a massless scalar particle is given by

$$
\square \psi=g^{-1 / 2} \partial_{\mu}\left[g^{1 / 2} g^{\mu \nu} \partial_{\nu}\right] \psi=M^{2} \psi .
$$

For the metric (8), the Klein-Gordon equation becomes

$$
\begin{aligned}
\frac{\partial^{2} \psi}{\partial t^{2}}= & -\left\{\frac{\partial^{2} \psi}{\partial r^{2}}+\frac{1}{r^{2} \alpha^{2}} \frac{\partial^{2} \psi}{\partial \theta^{2}}+\frac{1}{r^{2} \alpha^{2} \sin ^{2} \theta} \frac{\partial^{2} \psi}{\partial \varphi^{2}}\right. \\
& \left.+\frac{\cos \theta}{r^{2} \alpha^{2} \sin \theta} \frac{\partial \psi}{\partial \theta}+\frac{2}{r} \frac{\partial \psi}{\partial r}\right\} .
\end{aligned}
$$

In analogy with (10), the spatial operator $A$ is

$$
\begin{aligned}
A= & \left\{\frac{\partial^{2}}{\partial r^{2}}+\frac{1}{r^{2} \alpha^{2}} \frac{\partial^{2}}{\partial \theta^{2}}+\frac{1}{r^{2} \alpha^{2} \sin ^{2} \theta} \frac{\partial^{2}}{\partial \varphi^{2}}\right. \\
& \left.+\frac{\cos \theta}{r^{2} \alpha^{2} \sin \theta} \frac{\partial}{\partial \theta}+\frac{2}{r} \frac{\partial}{\partial r}\right\},
\end{aligned}
$$

and the equation to be solved is $\left(A^{*} \pm i\right) \psi=0$. Using separation of variables, $\psi=R(r) Y_{l}^{m}(\theta, \varphi)$, we get the radial portion of Eq. (14) as

$$
\frac{\mathrm{d}^{2} R(r)}{\mathrm{d} r^{2}}+\frac{2}{r} \frac{\mathrm{d} R(r)}{\mathrm{d} r}+\left(\frac{-l(l+1)}{r^{2} \alpha^{2}} \pm i\right) R(r)=0 .
$$

The square integrability of the above solution is checked by calculating the squared norm of the above solution in which the function space on each $t=$ constant hypersurface 
$\Sigma$ is defined as $\mathcal{H}=L^{2}(\Sigma, \mu)$ where $\mu$ is the measure given by the spatial metric volume element.

We easily recover the results showed in [9]: The spacetime of the global monopole remains singular from the point of view of relativistic quantum mechanics: the future of a given initial wave packet obeying the Klein-Gordon equation is not generally well determined, similarly to the future of a classical particle which reaches the classical singularity at $r=0$.

\subsection{Dirac fields}

The Newman-Penrose formalism will be used here to analyze a massless Dirac particle propagating in the space of global monopole. The signature of the metric (8) is changed to -2 in order to use the Dirac equation in Newman-Penrose formalism. Thus, the metric is given by

$\mathrm{d} s^{2}=\mathrm{d} t^{2}-\mathrm{d} r^{2}-r^{2} \alpha^{2}\left(\mathrm{~d} \theta^{2}+\sin ^{2} \theta \mathrm{d} \varphi^{2}\right)$.

The Chandrasekhar-Dirac (CD) [11] equations in the NewmanPenrose formalism are given by

$$
\begin{gathered}
(D+\epsilon-\rho) F_{1}+(\bar{\delta}+\pi-\alpha) F_{2}=0, \\
(\nabla+\mu-\gamma) F_{2}+(\delta+\beta-\tau) F_{1}=0, \\
(D+\bar{\epsilon}-\bar{\rho}) G_{2}-(\delta+\bar{\tau}-\bar{\alpha}) G_{1}=0, \\
(\nabla+\bar{\mu}-\bar{\gamma}) G_{1}-(\bar{\delta}+\bar{\beta}-\bar{\tau}) G_{2}=0,
\end{gathered}
$$

where $F_{1}, F_{2}, G_{1}$, and $G_{2}$ are the components of the wave function, $\epsilon, \rho, \pi, \alpha, \mu, \gamma, \beta$, and $\tau$ are the spin coefficients to be found and the "bar" denotes complex conjugation. The null tetrad vectors for the metric (19) are defined by

$$
\begin{aligned}
l^{a} & =(1,1,0,0), \\
n^{a} & =\left(\frac{1}{2},-\frac{1}{2}, 0,0\right), \\
m^{a} & =\frac{1}{\sqrt{2}}\left(0,0, \frac{1}{\alpha r}, \frac{i}{r \alpha \sin \theta}\right) .
\end{aligned}
$$

The directional derivatives in the Dirac equation are defined by $D=l^{a} \partial_{a}, \nabla=n^{a} \partial_{a}$ and $\delta=m^{a} \partial_{a}$. We define operators in the following way:

$\mathbf{D}_{0}=D$,

$\mathbf{D}_{0}^{\dagger}=-2 \nabla$,

$\mathbf{L}_{0}^{\dagger}=\sqrt{2} r \alpha \delta, \quad$ and $\quad \mathbf{L}_{1}^{\dagger}=\mathbf{L}_{0}^{\dagger}+\frac{\cot \theta}{2}$,

$\mathbf{L}_{0}=\sqrt{2} r \alpha \bar{\delta}$, and $\mathbf{L}_{1}=\mathbf{L}_{0}+\frac{\cot \theta}{2}$.

The nonzero spin coefficients are

$\mu=-\frac{1}{2 r}, \quad \rho=-\frac{1}{r}, \quad \beta=-\alpha=\frac{1}{2 \sqrt{2}} \frac{\cot \theta}{r \alpha}$.
Substituting nonzero spin coefficients and the definitions of the operators given above into the $\mathrm{CD}$ equations leads to

$$
\begin{aligned}
& \left(\mathbf{D}_{0}+\frac{1}{r}\right) F_{1}+\frac{1}{r \alpha \sqrt{2}} \mathbf{L}_{1} F_{2}=0, \\
& -\frac{1}{2}\left(\mathbf{D}_{0}^{\dagger}+\frac{1}{r}\right) F_{2}+\frac{1}{r \alpha \sqrt{2}} \mathbf{L}_{1}^{\dagger} F_{1}=0, \\
& \left(\mathbf{D}_{0}+\frac{1}{r}\right) G_{2}-\frac{1}{r \alpha \sqrt{2}} \mathbf{L}_{1}^{\dagger} G_{1}=0, \\
& \frac{1}{2}\left(\mathbf{D}_{0}^{\dagger}+\frac{1}{r}\right) G_{1}+\frac{1}{r \alpha \sqrt{2}} \mathbf{L}_{1} G_{2}=0 .
\end{aligned}
$$

For the solution of the $\mathrm{CD}$ equations, we assume a separable solution in the form of

$$
\begin{aligned}
F_{1} & =f_{1}(r) Y_{1}(\theta) \mathrm{e}^{i(k t+m \varphi)}, \\
F_{2} & =f_{2}(r) Y_{2}(\theta) \mathrm{e}^{i(k t+m \varphi)}, \\
G_{1} & =g_{1}(r) Y_{3}(\theta) \mathrm{e}^{i(k t+m \varphi)}, \\
G_{2} & =g_{2}(r) Y_{4}(\theta) \mathrm{e}^{i(k t+m \varphi)} .
\end{aligned}
$$

Here $\left\{f_{1}, f_{2}, g_{1}, g_{2}\right\}$ and $\left\{Y_{1}, Y_{2}, Y_{3}, Y_{4}\right\}$ are functions of $r$ and $\theta$, respectively, $m$ is the azimuthal quantum number, and $k$ is the frequency of the Dirac spinor, which is assumed to be positive and real. By substituting (25) in (24) we will see that with these assumptions

$f_{1}(r)=g_{2}(r) \quad$ and $\quad f_{2}(r)=g_{1}(r)$,

$Y_{1}(\theta)=Y_{3}(\theta)$ and $Y_{2}(\theta)=Y_{4}(\theta)$.

The Dirac equation reduces to two equations. The radial parts of the Dirac equations become

$$
\begin{aligned}
\left(\mathbf{D}_{0}+\frac{1}{r}\right) f_{1}(r) & =\frac{\lambda}{r \alpha \sqrt{2}} f_{2}(r), \\
\frac{1}{2}\left(\mathbf{D}_{0}^{\dagger}+\frac{1}{r}\right) f_{2}(r) & =\frac{\lambda}{r \alpha \sqrt{2}} f_{1}(r),
\end{aligned}
$$

where $\lambda$ comes from separation of the variables. We further assume that

$$
\begin{aligned}
& f_{1}(r)=\frac{\Psi_{1}(r)}{r}, \\
& f_{2}(r)=\frac{\sqrt{2} \Psi_{2}(r)}{r},
\end{aligned}
$$

then (28) transforms into

$\mathbf{D}_{0} \Psi_{1}=\frac{\lambda^{\prime}}{r} \Psi_{2}$

$\mathbf{D}_{0}^{\dagger} \Psi_{2}=\frac{\lambda^{\prime}}{r} \Psi_{1}$,

where $\lambda^{\prime}=\frac{\lambda}{\alpha}$, so we will have

$$
\begin{aligned}
& \left(\frac{\mathrm{d}}{\mathrm{d} r}+i k\right) \Psi_{1}=\frac{\lambda^{\prime}}{r} \Psi_{2}, \\
& \left(\frac{\mathrm{d}}{\mathrm{d} r}-i k\right) \Psi_{2}=\frac{\lambda^{\prime}}{r} \Psi_{1} .
\end{aligned}
$$


In order to write the above equation in a more compact form we combine the solutions in the following way:

$Z_{+}=\Psi_{1}+\Psi_{2}$,

$Z_{-}=\Psi_{2}-\Psi_{1}$.

After doing some calculations we end up with a pair of onedimensional Schrödinger-like wave equations with effective potentials,

$\left(\frac{\mathrm{d}^{2}}{\mathrm{~d} r^{2}}+k^{2}\right) Z_{ \pm}=V_{ \pm} Z_{ \pm}$

$V_{ \pm}=\frac{\lambda^{\prime 2}}{r^{2}} \mp \frac{\lambda^{\prime}}{r^{2}}$

In analogy with (10), the spatial operator $A$ for the massless case is

$A=-\frac{\mathrm{d}^{2}}{\mathrm{~d} r^{2}}+V_{ \pm}$

so we have [see (14)]

$$
\left(\frac{\mathrm{d}^{2}}{\mathrm{~d} r^{2}}-\left[\frac{\lambda^{\prime 2}}{r^{2}} \mp \frac{\lambda^{\prime}}{r^{2}}\right] \mp i\right) \psi_{ \pm}=0 \text {. }
$$

The solutions of the above equations are expressible using Bessel functions of the first and second kind in the following way:

$$
\begin{aligned}
\psi_{+}= & C_{1} \sqrt{r} J\left(\lambda^{\prime}-\frac{1}{2}, \frac{r}{\sqrt{2}}(1-i)\right) \\
& +C_{2} \sqrt{r} Y\left(\lambda^{\prime}-\frac{1}{2}, \frac{r}{\sqrt{2}}(1-i)\right), \\
\psi_{-}= & C_{1}^{\prime} \sqrt{r} J\left(\lambda^{\prime}+\frac{1}{2}, \frac{r}{\sqrt{2}}(1+i)\right) \\
& +C_{2}^{\prime} \sqrt{r} Y\left(\lambda^{\prime}+\frac{1}{2}, \frac{r}{\sqrt{2}}(1+i)\right) .
\end{aligned}
$$

Using the asymptotic formulas for Bessel functions when $r \rightarrow \infty\left(Y(\kappa, z) \approx z^{-1 / 2} \sin (z-\kappa \pi / 2-\pi / 4)\right.$ and $J(\kappa, z) \approx$ $\left.z^{-1 / 2} \cos (z-\kappa \pi / 2-\pi / 4)\right)$ and noting the complex argument in both solutions one can find a combination of constants $C_{1}, C_{2}$ or $C_{1}^{\prime}, C_{2}^{\prime}$ which is square integrable near infinity. (But it is also possible to choose the constants differently so that both solutions are not square integrable!)

When $r \rightarrow 0$ the approximate expressions for Bessel functions $\left(Y(\kappa, z) \approx z^{-\kappa}\right.$ for $\kappa \neq 0, Y(0, z) \approx \ln (z / 2)$ and $\left.J(\kappa, z) \approx z^{\kappa}\right)$ imply that for $C_{2}=0$ and $C_{2}^{\prime}=0$ we have square integrable solution near zero. (Here again if we suppose $C_{1}=0$ and $C_{1}^{\prime}=0$, for $\kappa \geq 3 / 2$, the solutions are not square integrable! One could restrict the analysis to only certain wave modes and purposely choose the modes to be quantum regular.)

But since we have a solution of equations valid on the whole domain (not just asymptotic forms of equations) we can match the behavior at zero and infinity. Based on the results we can have a solution square integrable over the whole domain and therefore our deficiency indices are nonzero. The operator is not essentially self-adjoint and the spacetime is quantum mechanically singular.

\section{Quantum gravity}

Now we are going to investigate the singularity of a general global monopole using techniques from loop quantization in the manner of [23]. Consider (2), for $r<\frac{2 G M}{1-8 \pi G \eta^{2}}$. This metric describes spacetime inside the horizon of a black hole. The coordinate $r$ is timelike and the coordinate $t$ is spatial there; for convenience we rename them as $r \equiv T$ and $t \equiv r$ with $T \in\left[0, \frac{2 G M}{1-8 \pi G \eta^{2}}\right]$ and $r \in[-\infty,+\infty]$, and the metric becomes

$$
\mathrm{d} s^{2}=-\left(\alpha^{2}-\frac{2 G M}{T}\right) \mathrm{d} r^{2}+\frac{\mathrm{d} T^{2}}{\left(\alpha^{2}-\frac{2 G M}{T}\right)}+T^{2} \mathrm{~d} \Omega^{2}
$$

we eliminate the coefficient of $\mathrm{d} T^{2}$ by defining a new temporal variable $\tau$ via

$$
\mathrm{d} \tau=\frac{\mathrm{d} T}{\sqrt{\frac{2 G M}{T}-\alpha^{2}}}
$$

Accordingly, the metric becomes

$$
\mathrm{d} s^{2}=-\mathrm{d} \tau^{2}+\left(\frac{2 G M}{T}-\alpha^{2}\right) \mathrm{d} r^{2}+T^{2}\left(\mathrm{~d} \theta^{2}+\sin ^{2} \theta \mathrm{d} \varphi^{2}\right) .
$$

We introduce two functions $a^{2}(\tau) \equiv \frac{2 G m}{T}-\alpha^{2}$ and $b^{2}(\tau) \equiv$ $T^{2}(\tau)$ and redefine $\tau \equiv t$. The metric becomes

$\mathrm{d} s^{2}=-\mathrm{d} t^{2}+a^{2}(t) \mathrm{d} r^{2}+b^{2}(t)\left(\mathrm{d} \theta^{2}+\sin ^{2} \theta \mathrm{d} \varphi^{2}\right)$

this metric describes a homogeneous, anisotropic KantowskiSachs cosmological model with a spatial section having the topology $\mathbf{R} \times \mathbf{S}^{2}$. From this observation comes the motivation to use the LQC approach. In our case $a(t)$ is a function of $b(t)$.

\subsection{Classical observables}

The corresponding action for gravity minimally coupled with a scalar field [described by (3)] can be written in the form

$$
\begin{aligned}
S= & \frac{1}{16 \pi G} \int \mathrm{d} t \mathrm{~d}^{3} x N h^{1 / 2}\left[K_{i j} K^{i j}-K^{2}\right. \\
& \left.+{ }^{(3)} R-\frac{16 \pi G \eta^{2}}{b^{2}}\right],
\end{aligned}
$$


and by considering the metric (38), the action becomes

$$
\begin{aligned}
S= & \frac{-1}{8 \pi G} \int \mathrm{d} t \int_{0}^{R} \mathrm{~d} r \int_{0}^{2 \pi} \mathrm{d} \phi \int_{0}^{\pi} \mathrm{d} \theta \sin \theta a b^{2} \\
& \times\left[\frac{\dot{b}^{2}}{b^{2}}+\frac{2 \dot{a} \dot{b}}{a b}-\frac{\alpha^{2}}{b^{2}}\right] .
\end{aligned}
$$

By using the relation between $a$ and $b$, we will be able to write the action in terms of a single function,

$$
\begin{aligned}
S= & \frac{R \alpha^{2}}{2 G} \int \mathrm{d} t \sqrt{\frac{b}{2 G M}}\left(1-\frac{\alpha^{2} b}{2 G M}\right)^{-1 / 2} \\
& \times\left[\dot{b}^{2}+\frac{2 G M}{b}\left(1-\frac{\alpha^{2} b}{2 G M}\right)\right] .
\end{aligned}
$$

Now, we will compute the Hamiltonian (Hamiltonian constraint). The momentum associated to the chosen configuration variable is

$p_{b}=\frac{R \alpha^{2} \dot{b}}{G} \sqrt{\frac{b}{2 G M}}\left(1-\frac{\alpha^{2} b}{2 G M}\right)^{-1 / 2}$,

and therefore we obtain

$$
\begin{aligned}
H & =p_{b} \dot{b}-L \\
& =\sqrt{\frac{2 G M}{b}} \sqrt{1-\frac{\alpha^{2} b}{2 G M}}\left[\frac{G p_{b}^{2}}{2 R \alpha^{2}}-\frac{R \alpha^{2}}{2 G}\right] .
\end{aligned}
$$

Now, we calculate the Hamiltonian constraint in terms of $\dot{b}$ :

$$
\begin{aligned}
H= & \frac{R \alpha^{2}}{2 G} \sqrt{\frac{b}{2 G M}}\left(1-\frac{\alpha^{2} b}{2 G M}\right)^{-1 / 2} \\
& \times\left[\dot{b}^{2}-\frac{2 G M}{b}\left(1-\frac{\alpha^{2} b}{2 G M}\right)\right]=0,
\end{aligned}
$$

and immediately we get the following solution:

$\dot{b}^{2}=\frac{2 G M}{b}-\alpha^{2}$,

which is exactly (36). When the radius of the horizon, $r_{h}=$ $\frac{2 G M}{\alpha^{2}}$, is much larger than the scale on which we are probing the singularity, we can write

$1-\frac{\alpha^{2} b}{2 G M} \sim 1$

so the Hamiltonian takes the form

$H=\sqrt{\frac{2 G M}{b}}\left[\frac{G P_{b}^{2}}{2 R \alpha^{2}}-\frac{R \alpha^{2}}{2 G}\right]$.

The spatial volume

$$
\begin{aligned}
V & =\int \mathrm{d} r \mathrm{~d} \theta \mathrm{d} \phi \sqrt{h}=4 \pi R a b^{2} \\
& =4 \pi R b^{3 / 2} \sqrt{2 G M} \sqrt{1-\frac{\alpha^{2} b}{2 G M}}
\end{aligned}
$$

simplifies when using the above approximation and we obtain

$$
\begin{aligned}
& V=l_{0} b^{3 / 2}, \\
& l_{0}=4 \pi R \sqrt{2 G M} .
\end{aligned}
$$

The canonical pair is given by $b \equiv x$ and $p_{b}$, with Poisson bracket $\left\{x, p_{b}\right\}=1$.

For isotropic models, only holonomies evaluated in isotropic connections $A_{a}^{i}=\tilde{c} \delta_{a}^{i}$ appear. Along straight lines in the direction of the translation symmetries $X_{I}^{a}=$ $\left(\partial / \partial X^{I}\right)^{a}$, the holonomies $\exp \left(\int X_{I}^{a} A_{a}^{i} \tau_{i}\right)$ from the fundamental representation of $S U$ (2) have matrix elements of the form $\exp (i \mu c)$, where $\mu$ depends on the length of the curve used. Here, it turns out to be useful to introduce $c:=V_{0}^{1 / 3} \tilde{c}$ defined in terms of the coordinate size $V_{0}$ of the region used to define the isotropic phase space [30].

Using this motivation we introduce the following function, which will be used instead of the momentum (from now on we leave out the subscript $b$ for the momentum associated with this observable) [23]:

$U_{\gamma}(p) \equiv \exp \left(8 \pi G \frac{i \gamma}{L} p\right)$

where $\gamma$ is a real parameter and $L$ fixes the length scale. The parameter $\gamma$ determines the separation of the momentum points in the phase space.

The pair $\left(x, U_{\gamma}(p)\right)$ has the following Poisson bracket algebra:

$$
\left\{x, U_{\gamma}(p)\right\}=8 \pi G \frac{i \gamma}{L} U_{\gamma}(p) .
$$

A straightforward calculation gives

$$
\begin{aligned}
U_{\gamma}^{-1}\left\{V^{n}, U_{\gamma}\right\} & =l_{0}^{n} U_{\gamma}^{-1}\left\{|x|^{3 n / 2}, U_{\gamma}\right\} \\
& =i 8 \pi G l_{0}^{n} \frac{\gamma}{L} \frac{3 n}{2} \operatorname{sgn}(x)|x|^{3 n / 2-1} .
\end{aligned}
$$

We are concerned with the quantity $\frac{1}{|x|}$, which can serve as an indicator for the presence of the singularity because classically it diverges for $|x| \rightarrow 0$, thus producing a singularity. From this moment we choose $n=1 / 3$

$$
\frac{\operatorname{sgn}(x)}{\sqrt{|x|}}=-\frac{2 L i}{8 \pi G l_{0}^{1 / 3} \gamma} U_{\gamma}^{-1}\left\{V^{1 / 3}, U_{\gamma}\right\} \text {. }
$$

\subsection{Quantization}

We will use the basis of Hilbert space introduced in [22, 23 ], which is formed by eigenstates of $\hat{x}$. This implies the existence of a self-adjoint operator $\hat{x}$, acting on the basis states according to

$\hat{x}|\boldsymbol{\mu}\rangle=L \mu|\boldsymbol{\mu}\rangle$.

Next, we want to promote the classical momentum function $U_{\gamma}=\mathrm{e}^{\left(8 \pi G \frac{i \gamma}{L} p\right)}$ to an operator (note that it is not exactly a 
holonomy of a connection but rather a translation generator). We can do so by defining the action of $\hat{U}_{\gamma}$ on the basis states with the help of the definition (53) and using a commutation relation based on the Poisson bracket between $x$ and $U_{\gamma}$. Applying the natural definition of $\hat{U}_{\gamma}$ as a translation operator $[24,30]$ and computing the commutation relation we obtain

$\hat{U}_{\gamma}|\boldsymbol{\mu}\rangle=|\boldsymbol{\mu}-\boldsymbol{\gamma}\rangle, \quad\left[\hat{x}, \hat{U}_{\gamma}\right]=-\gamma L \hat{U}_{\gamma}$.

Using canonical quantization of the Poison bracket $i \hbar\{,\} \rightarrow$ [, ], and (50), we get a relation for the length scale

$L=\sqrt{8 \pi} l_{p}$.

\subsection{Volume operator and disappearance of the singularity}

In the vicinity of the singularity we assume the approximate equation (48). Then the volume operator acts in the following way on the basis states:

$\hat{V}|\boldsymbol{\mu}\rangle=l_{0}|x|^{3 / 2}|\boldsymbol{\mu}\rangle=l_{0}|L \mu|^{3 / 2}|\boldsymbol{\mu}\rangle$.

Using (52) and promoting the Poisson brackets to commutators, while setting $\gamma=1$, we find

$$
\frac{\widehat{1}}{|x|}=\frac{1}{2 \pi l_{p}^{2} l_{0}^{2 / 3}}\left(\hat{U}_{\gamma}^{-1}\left[\hat{V}^{1 / 3}, \hat{U}_{\gamma}\right]\right)^{2} .
$$

On the basis states this operator acts in the following way:

$$
\begin{aligned}
\hat{U}_{\gamma}^{-1}\left[\hat{V}^{1 / 3}, \hat{U}_{\gamma}\right]|\boldsymbol{\mu}\rangle & =\left(\hat{U}_{\gamma}^{-1} \hat{V}^{1 / 3} \hat{U}_{\gamma}-\hat{U}_{\gamma}^{-1} \hat{U}_{\gamma} \hat{V}^{1 / 3}\right)|\boldsymbol{\mu}\rangle \\
& =l_{0}^{1 / 3} l_{p}^{1 / 2}(\sqrt{\mu-1}-\sqrt{\mu})|\boldsymbol{\mu}\rangle, \quad(58
\end{aligned}
$$

so finally we get

$\frac{\widehat{1}}{|x|}|\boldsymbol{\mu}\rangle=\sqrt{\frac{2}{\pi l_{p}^{2}}}(\sqrt{\mu-1}-\sqrt{\mu})^{2}|\boldsymbol{\mu}\rangle$.

We can see that the spectrum is bounded from above and so the singularity is resolved in the quantum theory (the theory gives finite predictions for observables related to a singularity). In fact, the eigenvalue of the operator $\frac{\widehat{1}}{|x|}$ corresponding to the state $|\mathbf{0}\rangle$, which probes the classical singularity, is equal to $\sqrt{\frac{2}{\pi l_{p}^{2}}}$, which is the highest eigenvalue of the spectrum. Specifically, the operator corresponding to the curvature invariant

$$
\begin{gathered}
\mathcal{R}_{\mu \nu \rho \sigma} \mathcal{R}^{\mu \nu \rho \sigma}=\frac{48 M^{2} G^{2}}{r^{6}}+\frac{128 M \pi G^{2} \eta^{2}}{r^{5}}+\frac{256 G^{2} \pi^{2} \eta^{4}}{r^{4}} \\
\equiv \frac{48 M^{2} G^{2}}{b(t)^{6}}+\frac{128 M \pi G^{2} \eta^{2}}{b(t)^{5}}+\frac{256 G^{2} \pi^{2} \eta^{4}}{b(t)^{4}}
\end{gathered}
$$

is then automatically finite in quantum mechanics. Promoting it to an operator and evaluating on $|\mathbf{0}\rangle$ we get

$$
\begin{aligned}
& \mathcal{R}_{\mu \nu \rho \sigma} \mathcal{R}^{\mu} v \rho \sigma|\mathbf{0}\rangle \\
& =\left(\frac{4 \sqrt{8 M^{2} G^{2}}}{|x|^{6}}+\frac{128 \widehat{M \pi G^{2}} \eta^{2}}{|x|^{5}}+\frac{25 \widehat{6 \pi^{2} G^{2}} \eta^{4}}{|x|^{4}}\right)|\mathbf{0}\rangle \\
& =\left(\frac{384 M^{2} G^{2}}{\pi^{3} l_{p}^{6}}+\sqrt{\frac{2}{\pi^{5}}} \frac{512 M \pi G^{2} \eta^{2}}{l_{p}^{5}}+\frac{1024 \pi^{2} G^{2} \eta^{4}}{\pi^{2} l_{p}^{4}}\right)|\mathbf{0}\rangle .
\end{aligned}
$$

On the other hand, when $|\mu| \rightarrow \infty$ the eigenvalue of $\frac{\widehat{1}}{|x|}$ goes to zero, which is a natural behavior for large $|x|$.

One should note that the above result comes from expressing the operator $\frac{\widehat{1}}{|x|}$ via (57). The resolution of the singularity is not given by the existence of a minimal length because the operator $\hat{x}$ contains zero in its (continuous) spectrum as can be seen from (53). The classical singularity is removed because $\frac{\widehat{1}}{|x|}$ has a finite eigenvalue even when the eigenvalue of $\hat{x}$ vanishes. This is in complete agreement with LQC. However, as pointed out in [31], the situation is more complicated when considering full Loop Quantum Gravity.

One should also make sure that the Kretschmann curvature scalar operator spectrum is finite at the classical singularity, which is, however, implied by its simple form for our model. The result (61) confirms both the resolution of the singularity (in contrast to classical behavior) and the vanishing of the curvature in the asymptotic region in the quantum picture (in agreement with classical behavior, as one would expect far from the singularity).

Also, it is possible to show that the quantum Hamiltonian constraint gives a discrete difference equation for the coefficients of the physical states.

\section{Conclusion}

We have seen that we have not been successful in removing the naked singularity by using relativistic quantum mechanics (for both Klein-Gordon and Dirac equations). On the other hand we have shown that the curvature singularity of a general global monopole is resolved when the geometry is quantized using loop techniques. Unfortunately, one cannot directly compare the results because the loop quantization relied on the radial coordinate being timelike beneath the horizon, which is not the case for a naked singularity of a pure monopole. But still, this might be an indication that the first method is not reliable for determining the fate of singularities in quantum theory and one should rather focus on quantization of the geometry itself. But even the approach inspired by loop quantization that relied on a restricted class of geometries should not be trusted completely. One should allow, e.g., for deviations from spherical symmetry to be completely sure about the fate of singularities. 
Acknowledgments T.T. gratefully acknowledges the hospitality of Institute of Theoretical Physics (Charles University in Prague) during her stay. O. S. was supported by Grant GAČR 14-37086G.

Open Access This article is distributed under the terms of the Creative Commons Attribution License which permits any use, distribution, and reproduction in any medium, provided the original author(s) and the source are credited.

Funded by $\mathrm{SCOAP}^{3}$ / License Version CC BY 4.0.

\section{Appendix: Geometric quantities}

The spatial metric is

$h_{i j}=\left(a^{2}(t), b^{2}(t), b^{2}(t) \sin ^{2} \theta\right)$.

The extrinsic curvature is $K_{i j}=-\frac{1}{2} \frac{\partial h_{i j}}{\partial t}$, and so

$K_{i j}=-\left(a \dot{a}, b \dot{b}, b \dot{b} \sin ^{2} \theta\right)$,

$K=K_{i j} h^{i j}=-\left(\frac{\dot{a}}{a}+2 \frac{\dot{b}}{b}\right)$,

$K_{i j} K^{i j}=\left(\frac{\dot{a}^{2}}{a^{2}}+2 \frac{\dot{b}^{2}}{b^{2}}\right)$

$K_{i j} K^{i j}-K^{2}=-2\left(\frac{\dot{b}^{2}}{b^{2}}+2 \frac{\dot{a} \dot{b}}{a b}\right)$.

The Ricci curvature for the spatial section is

${ }^{(3)} R=\frac{2}{b^{2}}$.

\section{References}

1. G.F.R. Ellis, B.G. Schmidt, Gen. Relativ. Gravit. 8, 915 (1977)

2. D.A. Konkowski, T.M. Helliwell, Gen. Relativ. Gravit. 33, 1131 (2001)

3. T.M. Helliwell, D.A. Konkowski, V. Arndt, Gen. Relativ. Gravit. 35, 79 (2003)
4. D.A. Konkowski, T.M. Helliwell, C. Wieland, Class. Quantum Gravity 21, 265 (2004)

5. D.A. Konkowski, C. Reese, T.M. Helliwell, C. Wieland, in Procedings of the Workshop on the Dynamics and Thermodynamics of Black holes and Naked Singularities, ed. L. Fatibene, M. Francaviglia, R. Giambo, G. Megli. Classical and Quantum Singularities of Levi-Civita Spacetimes with and without a Cosmological Constant (2004)

6. D.A. Konkowski, T.M. Helliwell, Int. J. Mod. Phys. A 26(22), 3878-3888 (2011)

7. J.P.M. Pitelli, P.S. Letelier, J. Math. Phys. 48, 092501 (2007)

8. J.P.M. Pitelli, P.S. Letelier, Phys. Rev. D 77, 124030 (2008)

9. J.P.M. Pitelli, P.S. Letelier, Phys. Rev. D 80, 104035 (2009)

10. P.S. Letelier, J.P.M. Pitelli, Phys. Rev. D 82, 104046 (2010)

11. S. Chandrasekhar, The Mathematical Theory of Black Holes (Oxford University Press, London, 1992)

12. O. Unver, O. Gurtug, Phys. Rev. D 82, 084016 (2010)

13. S. Habib Mazharimousavi, O. Gurtug, M. Halilsoy, Int. J. Mod. Phys. D 18, 2061-2082 (2009)

14. S. Habib Mazharimousavi, M. Halilsoy, I. Sakalli, O. Gurtug, Class. Quant. Gravity 27, 105005 (2010)

15. S. Habib Mazharimousavi, O. Gurtug, M. Halilsoy, O. Unver, Phys. Rev. D 84, 124021 (2011)

16. O. Gurtug, T. Tahamtan, Eur. Phys. J. C 72, 2091 (2012)

17. T.W.B. Kibble, Phys. A 9, 1387 (1976)

18. M. Barriola, A. Vilenkin, Phys. Rev. Lett. 63, 341-343 (1989)

19. E.R. Bezerra de Mello, Braz. J. Phys. 31, 211-222 (2001)

20. G.T. Horowitz, D. Marolf, Phys. Rev. D 52, 5670 (1995)

21. R.M. Wald, J. Math. Phys. (N.Y.) 21, 2082 (1980)

22. V. Husain, O. Winkler, Phys. Rev. D 69, 084016 (2003)

23. L. Modesto, Phys. Rev. D 70, 124009 (2004)

24. V. Husain, O. Winkler, Class. Quantum Gravity 22, L127-L133 (2005)

25. P.S. Letelier, Phys. Rev. D 20, 1294 (1979)

26. M. Reed, B. Simon, Functional Analysis (Academic Press, New York, 1980)

27. M. Reed, B. Simon, Fourier Analysis and Self-Adjointness (Academic Press, New York, 1975)

28. R.D. Richtmyer, Principles of Advanced Mathematical Physics (Springer, New York, 1978)

29. A. Ishibashi, A. Hosoya, Phys. Rev. D 60, 104028 (1999)

30. M. Bojowald, Canonical Gravity and Applications (Cambridge University Press, Cambridge, 2011)

31. T. Thiemann, Modern Canonical Quantum General Relativity (Cambridge University Press, Cambridge, 2007) 